\title{
Overexpression of Runt-Related Transcription Factor-2 Is Associated with Advanced Tumor Progression and Poor Prognosis in Epithelial Ovarian Cancer
}

\author{
Weiping Li, ${ }^{1}$ Shujuan Xu, ${ }^{2}$ Shuang Lin, ${ }^{3}$ and Wei Zhao ${ }^{4}$ \\ ${ }^{1}$ Department of Gynaecology and Obstetrics, Clinical Section, PLA General Hospital, Beijing 100853, China \\ ${ }^{2}$ Anesthesia and Operation Center, Clinical Section, PLA General Hospital, Beijing 100853, China \\ ${ }^{3}$ Department of Gynaecology and Obstetrics, Yantaishan Hosptial, Shandong, Yantai 264000, China \\ ${ }^{4}$ Clinical Section, PLA General Hospital, Beijing 100853, China \\ Correspondence should be addressed to Wei Zhao, zhao_wei301@163.com
}

Received 13 May 2012; Revised 4 August 2012; Accepted 4 August 2012

Academic Editor: Andre Van Wijnen

Copyright ( $) 2012$ Weiping Li et al. This is an open access article distributed under the Creative Commons Attribution License, which permits unrestricted use, distribution, and reproduction in any medium, provided the original work is properly cited.

\begin{abstract}
Aim. To investigate clinical significance of runt-related transcription factor (RUNX)-2 in epithelial ovarian cancer (EOC). Methods. RUNX2 protein expression and its subcellular localization were detected by immunohistochemistry in 116 patients with EOC. Results. RUNX2 protein was predominantly expressed in cell nucleus of EOC tissues. The expression level of RUNX2 in EOC tissues was significantly higher than that in normal ovarian tissues $(P<0.001)$. In addition, the nuclear labeling index (LI) of RUNX2 in tumor cells was significantly associated with the advanced clinical stage of EOC tissues $(P=0.001)$. Moreover, EOC patients with high RUNX2 LI had significantly shorter overall $(P<0.001)$ and progression-free $(P=0.002)$ survival than those with low RUNX2 LI. Especially, subgroup analysis revealed that EOC patients with high clinical stages (III IV) in high RUNX2 expression group demonstrated a significantly worse clinical outcome than those in low RUNX2 expression group, but patients with low clinical stages (I II) had no significantly different prognosis between high and low RUNX2 expression groups. Conclusions. Our data suggest for the first time that RUNX2 overexpression is associated with advanced tumor progression and poor clinical outcome of EOC patients. RUNX2 might be a novel prognostic marker of EOC.
\end{abstract}

\section{Introduction}

Epithelial ovarian cancer (EOC) represents one of the most common gynecologic malignancies worldwide. It has the highest mortality rate among malignant tumors in female reproductive system [1, 2]. Because of the lack of specific early symptoms or effective tumor biomarkers, most patients with EOC are diagnosed at the advanced stages, and the prognosis of these patients is still poor, even though there has been great improvement on traditional treatments, such as surgery, supplemented with radiotherapy and chemotherapy. The 5-year survival rate for EOC patients is only $30 \sim 40 \%$ [3]. Similar with other human malignancies, tumorigenesis and tumor progression of EOC are caused by numerous reproductive, environmental, and genetic risk factors. Therefore, it is of great importance to discover and analyze the genetic changes and molecular events involving the initiation, progression, and metastasis of EOC.

Mammalian runt-related transcription factor (RUNX) family contains three members (RUNX1 3) which form the core-binding factor $(\mathrm{CBF})$ complex and bind DNA to either activate or repress gene transcription [4]. As part of the CBF complex, the RUNX proteins are involved in the regulation of differentiation, survival, and growth in a variety of tissues [5]. Especially, their functions have high specificity. RUNX1 and RUNX2 are specifically essential for multiple haematopoietic lineages and osteogenesis, respectively, and RUNX3 is closely related with neurogenesis and gut development [6]. Accumulating studies have reported the oncogenic and tumor suppressive functions of the RUNX members. In the present study, we have chosen to focus on the role of RUNX2 in neoplastic disease. Because RUNX2 plays a pivotal 
role in the process of bone formation or osteogenesis [7], it is no doubt that its deregulation is strongly associated with the development of osteosarcoma [8]. In addition, the overexpression of RUNX2 has also been identified in several human malignancies, including prostate cancer [9], breast cancer [10], pancreatic cancer [11], colon carcinoma [12], and thyroid cancer [13]. Its carcinogenic properties have been further highlighted in the recent studies. For example, Tandon et al. [14] indicated that RUNX2 is a potential therapeutic target to block tumor suppressor gene silencing in lung cancer cells. Pratap et al. [10] found that the expression of RUNX2 in breast cancer cells may be important for tumor cell invasion through matrigel chambers. The results of Chua group revealed that RUNX2 nuclear staining was correlated with increased prostate-specific antigen (PSA) levels, higher Gleason scores and tumours with metastatic capability [9]. Sase et al. [12] identified RUNX2 as a potent prognostic factor in colon carcinoma patients through the promotion of cell proliferation and invasion properties. However, the expression patterns and involvement of RUNX2 in EOC are still unclear. Therefore, the aim of this study was to investigate the clinical significance of RUNX2 expression in EOC.

\section{Materials and Methods}

2.1. Patients and Tissue Samples. The study was approved by the Research Ethics Committee of General Hospital of PLA, China. Informed consent was obtained from all of the patients. All specimens were handled and made anonymous according to the ethical and legal standards.

We collected formalin-fixed, paraffin-embedded tissues from the primary ovaries obtained during surgery from women with EOC $(n=116)$. As controls, we also obtained normal ovarian tissues $(n=5)$ from women who underwent hysterectomies for benign disease. All operations were performed in the Department of Obstetrics and Gynecology at General Hospital of PLA from January 2005 to December 2006. All patients with only gynecology tumor were treated without preoperative radiotherapy, chemotherapy, or hormonal therapy. Surgical staging was established according to the International Federation of Gynecology and Obstetrics (FIGO) system. Debulking status was defined according to the size of the nodules left in the peritoneal cavity after surgery. The clinical features of 116 EOC patients were summarized in Table 1.

2.2. Immunohistochemistry Analysis. The specimens were fixed in $10 \%$ neutral buffered formalin and subsequently embedded with paraffin. The paraffin-embedded tissues were cut at $3 \mu \mathrm{m}$ and then deparaffinized with xylene and rehydrated for further hematoxylin eosin (H\&E) or 3,3' diaminobenzidine (DAB) immunohistochemistry staining employing DAKO EnVision System (Dako Diagnostics, Zug, Switzerland). Briefly, following treatment with 10\% normal goat serum for 45 min to block nonspecific binding sites, the consecutive tissue sections were incubated for $180 \mathrm{~min}$ at $25^{\circ} \mathrm{C}$ with mouse monoclonal antibody for human RUNX2 (dilution $1: 1000$, Abnova Corporation, Taipei, Taiwan). The specificity of the primary antibody has been validated by the previous studies $[15,16]$. After washing, peroxidase-labeled polymer and substrate-chromogen were then employed in order to visualize the staining of the interested proteins.

In tumor cells of EOC tissues, RUNX2 immunoreactivity was detected in the nucleus, and the immunoreactivity was evaluated as a labeling index (LI) according to the methods of previous studies $[15,16]$. Briefly, RUNX2 immunoreactivity was evaluated in the nuclei of more than 1000 tumor cells for each case, and LI was calculated as the percentage of RUNX2 positive cells per 1000 tumor cells counted at random in each section. This counting was performed under a $\times 400$ magnification.

2.3. Statistical Analysis. The software of SPSS version 12.0 for Windows (SPSS Inc., IL, USA) and SAS 9.1 (SAS Institute, Cary, NC, USA) were used for statistical analysis. The Pearson's chi-square and Fisher's exact tests were used to assess the statistical significance of the association between RUNX2 expression and clinicopathologic parameters. Kaplan-Meier curves were plotted to assess the effects of RUNX2 expression on overall and progression-free survival. Survival curves were compared using the log-rank test. Cox proportional hazard models were used to assess the prognostic significance of RUNX2 expression and several clinicopathologic parameters. Differences were considered statistically significant when $P$ value was less than 0.05 .

\section{Results}

3.1. Expression and Localization of RUNX2 in EOC Tissues. The expression patterns and cellular localization of RUNX2 in 116 EOC and 5 normal ovarian tissues were assessed by immunohistochemical analysis. As shown in Figure 1, RUNX2 immunoreactivity was predominantly localized in the nuclei of EOC cells (Figure 1(a)), while almost negligible in normal ovarian tissues (Figure 1(b)). The mean value of the RUNX2 LI in 116 EOC tissues detected was 56.3\% (range, 0-99\%), which was significantly higher than that in normal ovarian tissues (11.7\%; range, $0-35.2 \% ; P<0.001)$.

The median value of RUNX2 LI was $55.1 \%$. All the EOC tissues $(n=116)$ were divided into two groups: high RUNX2 expression group (RUNX2 LI $\geq 55.1 \%, n=78$ ) and low RUNX2 expression group (RUNX2 LI $<55.1 \%, n=38$ ).

3.2. Association of RUNX2 Expression with Clinicopathological Features of EOC Tissues. Table 1 summarized the association of RUNX2 expression with various clinicopathological features of EOC tissues. The nuclear LI of RUNX2 in tumor cells was significantly associated with the clinical stage of EOC tissues $(P=0.001)$. The EOC tissues with advanced clinical stage (III IV) more frequently showed high RUNX2 expression than those with low clinical stage (I II). However, RUNX2 expression was not correlated with age, grade, histological type, and residual tumor after surgery (all $P>$ 0.05). 
TABLE 1: Association of RUNX2 expression with clinicopathological features of epithelial ovarian cancer tissues.

\begin{tabular}{|c|c|c|c|c|}
\hline \multirow{2}{*}{ Features } & \multirow{2}{*}{ No. of patients } & \multicolumn{2}{|c|}{ RUNX2 expression $(n, \%)$} & \multirow[t]{2}{*}{$P$} \\
\hline & & Low & High & \\
\hline \multicolumn{5}{|l|}{ Age } \\
\hline$<50$ & 48 & $16(33.3)$ & $32(66.7)$ & \multirow{2}{*}{0.69} \\
\hline$\geq 50$ & 68 & $22(32.4)$ & $46(67.6)$ & \\
\hline \multicolumn{5}{|l|}{ Clinial stage } \\
\hline $\mathrm{I} \sim \mathrm{II}$ & 26 & $18(69.2)$ & $8(30.8)$ & \multirow{2}{*}{0.001} \\
\hline $\mathrm{III} \sim \mathrm{IV}$ & 90 & $20(22.2)$ & $70(77.8)$ & \\
\hline \multicolumn{5}{|c|}{ Pathological grade } \\
\hline $1 \sim 2$ & 33 & $15(45.5)$ & $18(54.5)$ & \multirow{2}{*}{0.06} \\
\hline 3 & 83 & $23(27.7)$ & $60(72.3)$ & \\
\hline \multicolumn{5}{|c|}{ Histological type } \\
\hline Serous & 88 & $28(31.2)$ & $60(68.8)$ & \multirow{2}{*}{0.57} \\
\hline Non-serous & 28 & $10(35.7)$ & $18(64.3)$ & \\
\hline \multicolumn{5}{|c|}{ Residual tumor after surgery } \\
\hline$<1 \mathrm{~cm}$ & 66 & $23(34.8)$ & $43(65.2)$ & \multirow{2}{*}{0.51} \\
\hline$\geq 1 \mathrm{~cm}$ & 50 & $15(30.0)$ & $35(70.0)$ & \\
\hline
\end{tabular}

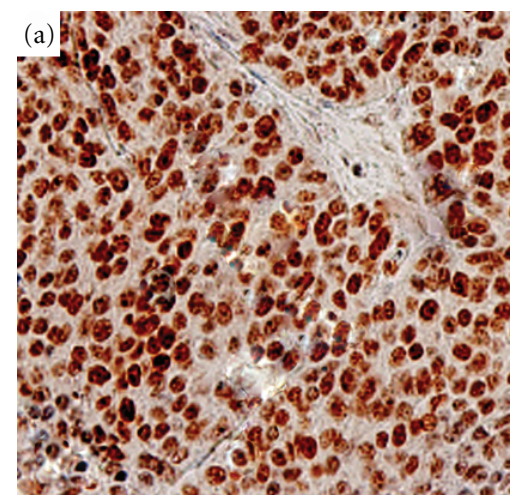

(a)

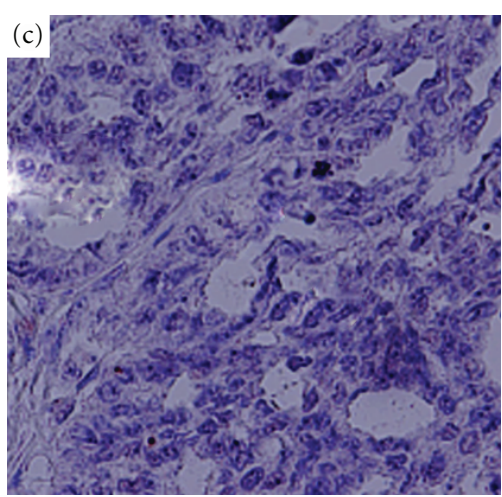

(c)

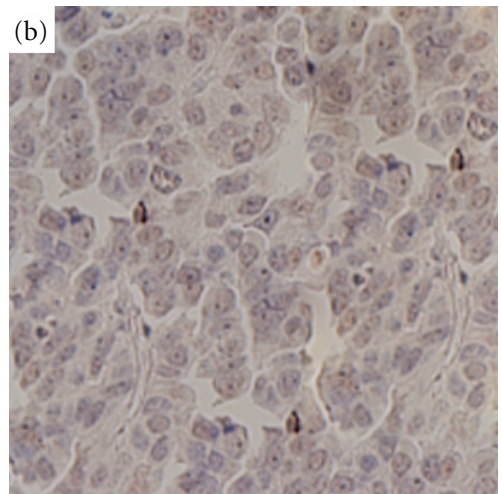

(b)

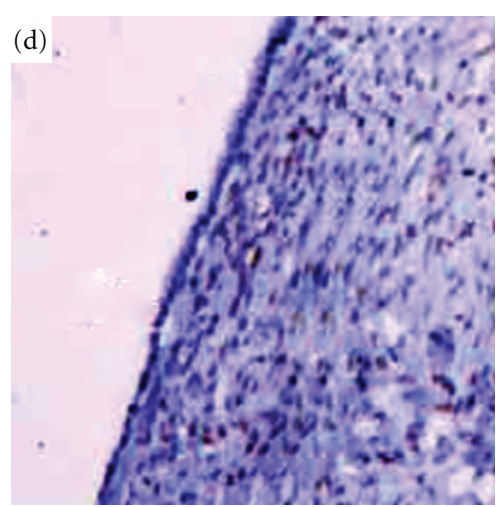

(d)

FIGURE 1: Immunohistochemical staining for RUNX2 in epithelial ovarian cancer and normal ovarian tissues (original magnification $\times 200$ ). (a) High RUNX2 expression ( $\mathrm{LI}=96.5 \%$ ) in epithelial ovarian cancer tissues with clinical stage IV. (b) Low RUNX2 expression (LI = 16.6\%) in epithelial ovarian cancer tissues with clinical stage I. RUNX2 immunoreactivity was predominantly localized in the nuclei of epithelial ovarian cancer cells. (c) Negative control without the primary antibody in epithelial ovarian cancer tissues. (d) Negative RUNX2 expression in normal ovarian tissues. 


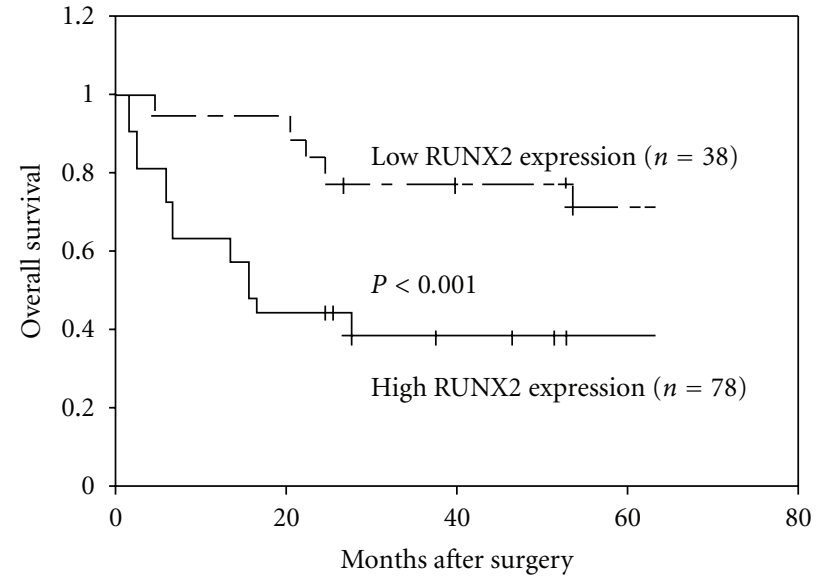

(a)

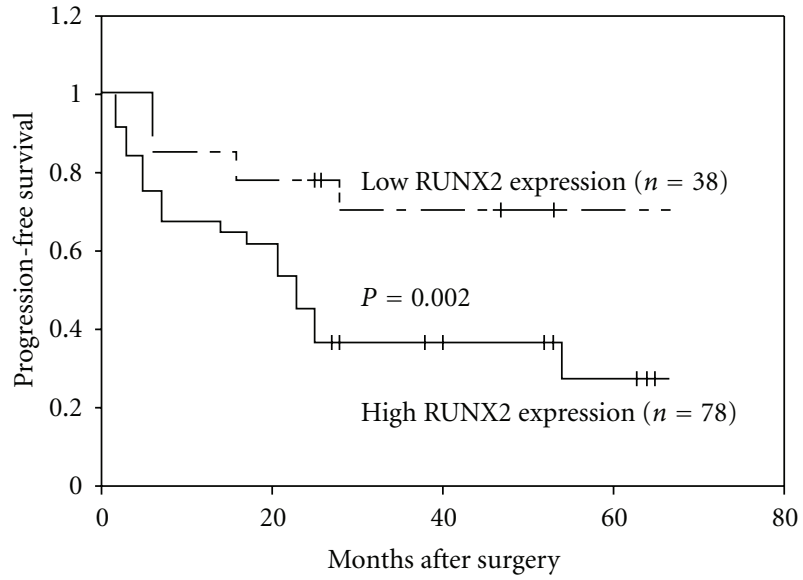

(b)

FIGURE 2: Kaplan-Meier overall (a) and progression-free (b) survival curves for epithelial ovarian cancer patients with high and low RUNX2 expression. Epithelial ovarian cancer patients with high RUNX2 expression had significantly shorter overall $(P<0.001)$ and progression-free $(P=0.002)$ survival than those with low RUNX2 expression did.

TABLE 2: Univariate analysis: factors predicting overall and progression-free survival.

\begin{tabular}{lcccc}
\hline \multirow{2}{*}{ Characteristic } & \multicolumn{2}{c}{ Overall survival } & \multicolumn{2}{c}{ Progression-free survival } \\
& $P$ value & Hazard ratio $(95 \%$ CI $)$ & $P$ value & Hazard ratio (95\% CI) \\
\hline Age & 0.16 & $1.582(0.832 \sim 3.168)$ & 0.27 & $1.001(0.739 \sim 2.802)$ \\
Clinical stage & $<0.001$ & $10.859(1.942 \sim 26.919)$ & $\mathbf{0 . 0 0 8}$ & $7.736(1.219 \sim 19.573)$ \\
Pathological grade & 0.06 & $4.266(1.031 \sim 10.533)$ & 0.09 & $3.152(1.006 \sim 8.131)$ \\
Histological type & 0.05 & $6.852(1.062 \sim 11.781)$ & 0.08 & $4.026(1.022 \sim 9.781)$ \\
Residual tumor after surgery & 0.11 & $3.049(1.001 \sim 7.103)$ & 0.25 & $2.892(0.903 \sim 6.098)$ \\
RUNX2 expression & $<0.001$ & $12.488(1.735 \sim 33.026)$ & $\mathbf{0 . 0 0 2}$ & $9.018(1.834 \sim 26.098)$ \\
\hline
\end{tabular}

3.3. Prognostic Implications of RUNX2 Expression in EOC. In order to investigate the prognostic implications of RUNX2 expression in overall survival and progression-free survival of EOC, the detailed clinical information of all 116 EOC patients in high RUNX2 expression and low RUNX2 expression groups was reviewed. Median follow-up time was 66.8 months (range, 2.2-118.9 months; mean, 66.1 months). At last followup, $73(62.9 \%)$ relapsed with a median time of 22.1 months (range, 2.8-85.2 months). As determined by the log-rank test, EOC patients with high RUNX2 LI had significantly shorter overall $(P<0.001$, Figure $2(a))$ and progression-free $(P=0.002$, Figure $2(\mathrm{~b}))$ survival than those with low RUNX2 LI did. Moreover, the univariate analysis revealed that both the advanced stage $(P<0.001$ and $P=$ 0.008 , resp. $)$ and the high RUNX2 expression $(P<0.001$ and $P=0.002$, resp.) predicted poorer overall and progressionfree survival of EOC patients (Table 2). Furthermore, the multivariate analyses identified the clinical stage $(P=0.01$ and $P=0.03$, resp.) and the RUNX2 LI (both $P=0.01$ ) in EOC cells as independent prognostic factors for overall and progression-free survival (Table 3 ).

Interestingly, subgroup analyses according to clinical stage revealed that EOC patients with high clinical stages (III IV) in high RUNX2 expression group demonstrated a significantly worse clinical outcome than those in low
RUNX2 expression group (Figures 3(a) and 3(b)), but patients with low clinical stages (I II) had no significantly different prognosis between high and low RUNX2 expression groups (Figures $3(\mathrm{c})$ and $3(\mathrm{~d})$ ).

\section{Discussion}

As the first leading cause of cancer death in female reproductive system malignant tumors, EOC has no characteristic early symptoms or tumor markers, leading to disappointing clinical outcome. Overall survival rates remain poor despite improvements in response rates. The clinical course of remission and relapse is commonly seen in patients undergoing therapy for EOC. Discovery and analysis of the genetic changes and molecular events have contributed largely to a better understanding of the molecular mechanisms of cancer ontogenesis. Accordingly, it is of great significance to identify novel specific diagnostic or prognostic markers that contribute to progression and metastasis of EOC. In the present study, we detected the overexpression of RUNX2 in EOC tissues compared with normal ovarian tissues, and its upregulation was closely related with the clinical stage and poor prognosis of EOC patients, which led us to believe that RUNX2 might be used as a candidate biomarker for aggressive disease behavior. To the best of our knowledge, 
TABLE 3: Multivariate analysis: factors predicting overall and progression-free survival.

\begin{tabular}{lcccc}
\hline \multirow{2}{*}{ Characteristic } & \multicolumn{2}{c}{ Overall survival } & \multicolumn{2}{c}{ Progression-free survival } \\
& $P$ value & Hazard ratio $(95 \%$ CI $)$ & $P$ value & 0.56 \\
\hline Age & 0.31 & $0.791(0.008 \sim 2.937)$ & $\mathbf{0 . 0 3}$ & $0.601(0.739 \sim 2.802)$ \\
Clinical stage & 0.01 & $7.133(1.028 \sim 22.160)$ & 0.1 & $6.698(1.011 \sim 20.287)$ \\
Pathological grade & 0.09 & $3.128(1.001 \sim 8.238)$ & 0.1 & $3.052(1.006 \sim 7.916)$ \\
Histological type & 0.08 & $3.161(1.067 \sim 8.650)$ & 0.37 & $3.033(1.002 \sim 7.823)$ \\
Residual tumor after surgery & 0.23 & $1.257(0.119 \sim 2.589)$ & $\mathbf{0 . 0 1}$ & $1.029(0.091 \sim 2.482)$ \\
RUNX2 expression & 0.01 & $7.338(1.263 \sim 22.893)$ & $7.018(1.238 \sim 22.037)$ \\
\hline
\end{tabular}

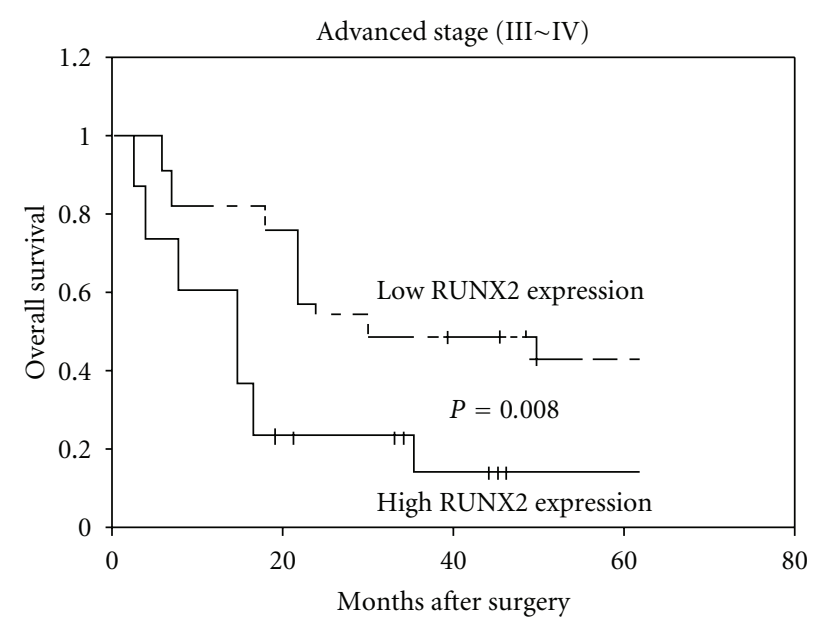

(a)

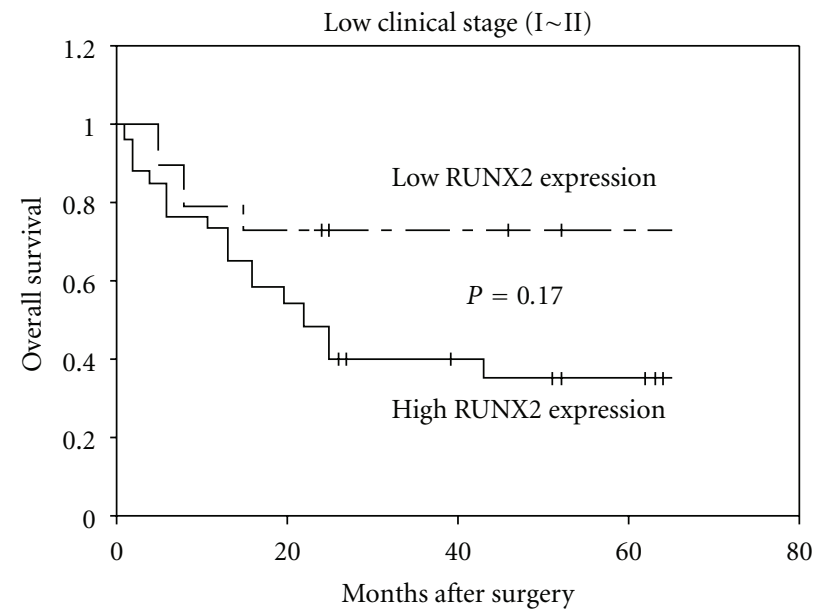

(c)

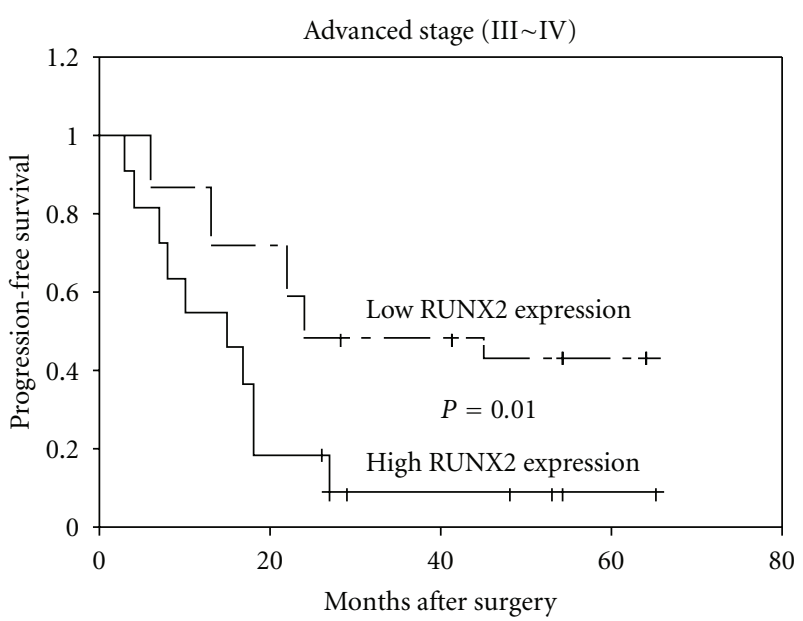

(b)

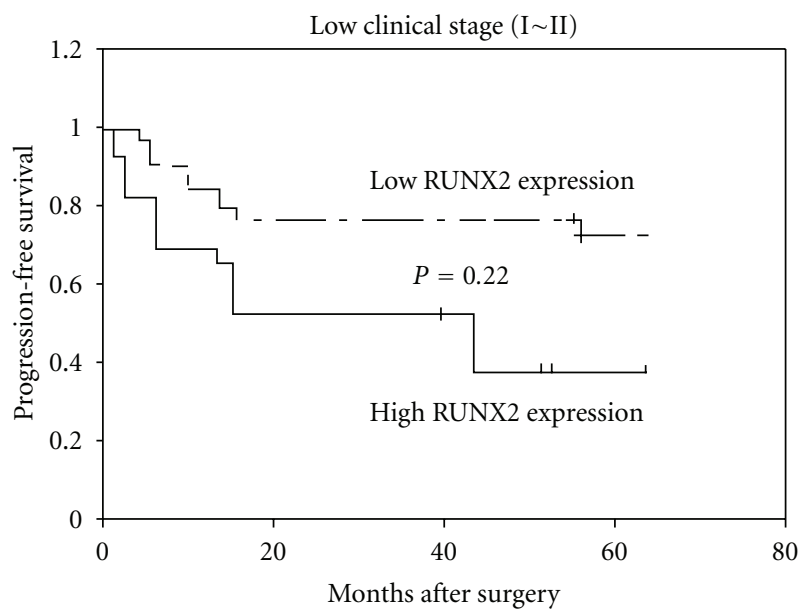

(d)

FIGURE 3: Kaplan-Meier overall (a and c) and progression-free (b and d) survival curves for epithelial ovarian cancer patients with different clinical stages. Subgroup analyses according to clinical stage revealed that epithelial ovarian cancer patients with high clinical stages (III $\sim$ IV) in high RUNX2 expression group demonstrated a significantly worse clinical outcome than those in low RUNX2 expression group (a and b), but patients with low clinical stages (I II) had no significantly different prognosis between high and low RUNX2 expression groups (c and $\mathrm{d})$.

this is the first investigation on the involvement of RUNX2 in EOC.

RUNX proteins have been demonstrated to play positive and negative roles in carcinogenesis according to different cancer types [17]. As a member of RUNXs, RUNX2, also known as core-binding factor, runt domain, a-subunit 1, CBFA1, AML3, or OSF2, is a lineage-specific transcription factor and the human homologue of mouse PEBP2A [18]. During embryonic development, RUNX2 is involved in the process of osteogenesis [19]. After birth, RUNX2 controls 
bone matrix deposition, especially collagen I, by differentiated osteoblasts [20]. Targeted disruption of RUNX2 in mice results in failure of osteoblast differentiation and bone formation, and RUNX2 haploinsufficiency in humans leads to the skeletal disorder cleidocranial dysplasia, with a similar phenotype observed in RUNX2 haploinsufficient mice [21]. In addition, very recently, Park et al. reported that RUNX2 transcription factor may be involved in various aspects of luteal function by directly regulating the expression of diverse luteal genes in luteinizing granulosa cells of rat ovaries [22]. In carcinogenesis, RUNX2 acts as a master regulator of tumor invasion and metastasis. High expression of RUNX2 is significantly related to metastasis of osteosarcoma [8]. Nuclear RUNX2 is increased in malignant versus benign prostate tissue and is associated with tumor aggression in general and metastasis in particular [9]. RUNX2 has the strongest basal expression among the RUNXs, and a significant increase in RUNX2 in carcinoma samples contributes to tumorigenesis in esophageal squamous cell carcinoma [23]. The differential expression of RUNX2 has been found between invasive, and noninvasive breast cancer cells, and its upregulation is linked to the invasive phenotype of cancer cells [10]. RUNX2 also plays an important role in angiogenesis by enhancing the endothelial cell proliferation, invasion and tube formation as well as indirectly by activating the promoter region of the vascular endothelial growth factor gene [24]. Edvardsson et al. [25] conducted genome-wide expression studies in combination with gene-pathway analyses and cross-correlation to estrogen receptor- $\beta$ (ER $\beta$-) chromatin-binding sites, and demonstrated that RUNX2 expression was induced by ER $\beta$ in human colorectal carcinoma cells. In the present study, our data found that RUNX2 significantly overexpressed in EOC tissues in contrast to normal ovarian tissues. Its expression status in EOC tissues was similar with another RUNX member, RUNX3 [26]. We also showed that the EOC tissues with advanced clinical stage more frequently tend to express high RUNX2. These findings provide evidence that the up-regulation of RUNX2 expression might be important in the carcinogenesis of EOC.

Because of the poor prognosis in EOC patients, our study also focused on the prognostic implications of RUNX2 for this cancer. Our survival analysis demonstrated that EOC patients whose tumors overexpressed RUNX2 had shorter overall and progression-free survival times than those with low RUNX2 expression tumors, especially those at advanced clinical stages. More importantly, further analysis using the Cox regression model confirmed that the RUNX2 expression was an independent factor in predicting overall and progression-free survival times for EOC patients. These findings provide evidence that RUNX2 could be regarded as a biomarker for predicting the outcome of EOC patients.

The function of RUNX2 is related with several molecules and multiple pathways, such as angiogenic factor, matrix metalloproteinases (MMPs), transcription factors, the MAPK pathway, the STAT pathway and the PI3K pathway [27], which are involved in ovarian carcinogenesis. For example, Duan et al. [28] indicated that the abnormal activation on PI3 K/PKB signaling pathway may be correlated with the occurrence and development of EOC; Chakrabarty and Kondratick [29] also suggested that activation on multiple cascades of the MAPK pathway may promote the invasion and metastasis of EOC. In this study, although our results demonstrate the aberrant expression and important clinical significance of RUNX2 in EOC patients, the exact mechanism of RUNX2 upregulation in EOC is still not clearly understood. In this context, further studies are needed to determine the molecular mechanism of RUNX2 dysfunction in human ovarian carcinogenesis.

In conclusion, our data suggest for the first time that RUNX2 overexpression is associated with advanced tumor progression and poor clinical outcome of EOC patients. RUNX2 might be a novel prognostic marker of EOC.

\section{Conflict of Interests}

The authors declare that they have no conflict of interests.

\section{References}

[1] S. J. Jordan, K. L. Cushing-Haugen, K. G. Wicklund, J. A. Doherty, and M. A. Rossing, "Breast-feeding and risk of epithelial ovarian cancer," Cancer Causes and Control, vol. 21, no. 1, pp. 109-116, 2010.

[2] M. Rota, E. Pasquali, L. Scotti et al., "Alcohol drinking and epithelial ovarian cancer risk. A systematic review and metaanalysis," Gynecologic Oncology, vol. 125, no. 3, pp. 758-763, 2012.

[3] M. Wang, Y. He, L. Shi, and C. Shi, "Multivariate analysis by Cox proportional hazard model on prognosis of patient with epithelial ovarian cancer," European Journal of Gynaecological Oncology, vol. 32, no. 2, pp. 171-177, 2011.

[4] N. O. Chimge, S. K. Baniwal, G. H. Little et al., "Regulation of breast cancer metastasis by RUNX2 and estrogen signaling: the role of SNAI2," Breast Cancer Research, vol. 13, article R127, 2011.

[5] M. L. Slattery, A. Lundgreen, J. S. Herrick, B. J. Caan, J. D. Potter, and R. K. Wolff, "Associations between genetic variation in RUNX1, RUNX2, RUNX3, MAPK1 and eIF4E and risk of colon and rectal cancer: additional support for a TGF$\beta$-signaling pathway," Carcinogenesis, vol. 32, no. 3, pp. 318326, 2011.

[6] G. H. Little, H. Noushmehr, S. K. Baniwal, B. P. Berman, G. A. Coetzee, and B. Frenkel, "Genome-wide RUNX2 occupancy in prostate cancer cells suggests a role in regulating secretion," Nucleic Acids Research, vol. 40, pp. 3538-3547, 2012.

[7] J. W. Martin, M. Zielenska, G. S. Stein, A. J. van Wijnen, and J. A. Squire, "The role of RUNX2 in osteosarcoma oncogenesis," Sarcoma, vol. 2011, Article ID 282745, 13 pages, 2011.

[8] M. van der Deen, J. Akech, D. Lapointe et al., "Genomic promoter occupancy of runt-related transcription factor RUNX2 in Osteosarcoma cells identifies genes involved in cell adhesion and motility," The Journal of Biological Chemistry, vol. 287, pp. 4503-4517, 2012.

[9] C. W. Chua, Y. T. Chiu, H. F. Yuen et al., "Suppression of androgen-independent prostate cancer cell aggressiveness by FTY720: validating Runx2 as a potential antimetastatic drug screening platform," Clinical Cancer Research, vol. 15, no. 13, pp. 4322-4335, 2009.

[10] J. Pratap, A. Javed, L. R. Languino et al., "The Runx2 osteogenic transcription factor regulates matrix metalloproteinase 9 in bone metastatic cancer cells and controls cell invasion," 
Molecular and Cellular Biology, vol. 25, no. 19, pp. 8581-8591, 2005.

[11] H. Kayed, X. Jiang, S. Keleg et al., "Regulation and functional role of the Runt-related transcription factor-2 in pancreatic cancer," British Journal of Cancer, vol. 97, no. 8, pp. 1106-1115, 2007.

[12] T. Sase, T. Suzuki, K. Miura et al., "Runt-related transcription factor 2 in human colon carcinoma: a potent prognostic factor associated with estrogenreceptor," International Journal of Cancer. In press.

[13] L. Dalle Carbonare, A. Frigo, G. Francia et al., "Runx2 mRNA expression in the tissue, serum, and circulating nonhematopoietic cells of patients with thyroid cancer," The Journal of Clinical Endocrinology \& Metabolism, vol. 97, no. 7, pp. E1249-E1256, 2012.

[14] M. Tandon, K. Gokul, S. A. Ali et al., "RUNX2 mediates epigenetic silencing of the bone morphogenetic protein-3B (BMP-3B/GDF10) in lung cancer cells," Molecular Cancer, vol. 11, article 27, 2012.

[15] K. Y. Won, H. R. Park, and Y. K. Park, "Prognostic implication of immunohistochemical Runx2 expression in osteosarcoma," Tumori, vol. 95, no. 3, pp. 311-316, 2009.

[16] Y. Onodera, Y. Miki, T. Suzuki et al., "Runx2 in human breast carcinoma: its potential roles in cancer progression," Cancer Science, vol. 101, no. 12, pp. 2670-2675, 2010.

[17] K. Blyth, F. Vaillant, A. Jenkins et al., "Runx2 in normal tissues and cancer cells: a developing story," Blood Cells, Molecules, and Diseases, vol. 45, no. 2, pp. 117-123, 2010.

[18] D. J. Purcell, O. Khalid, C. Y. Ou et al., "Recruitment of coregulator G9a by RUNX2 for selective enhancement or suppression of transcription," Journal of Cellular Biochemistry, vol. 113, no. 7, pp. 2406-2414, 2012.

[19] K. F. Underwood, D. R. D’Souza, M. Mochin-Peters et al., "Regulation of RUNX2 transcription factor-DNA interactions and cell proliferation by vitamin D3 (cholecalciferol) prohormone activity," Journal of Bone and Mineral Research, vol. 27, pp. 913-925, 2012.

[20] K. N. Lee, W. G. Jang, E. J. Kim et al., “Orphan nuclear receptor COUP-TFII negatively regulates BMP2-induced osteoblast differentiation through suppressing RUNX2 activity," The Journal of Biological Chemistry, vol. 287, no. 23, pp. 1888818899, 2012.

[21] Y. Li, C. Ge, J. P. Long et al., "Biomechanical stimulation of osteoblast gene expression requires phosphorylation of the RUNX2 transcription factor," Journal of Bone and Mineral Research, vol. 27, no. 6, pp. 1263-1274, 2012.

[22] E. S. Park, A. K. Lind, P. Dahm-Kähler et al., "RUNX2 transcription factor regulates gene expression in luteinizing granulosa cells of rat ovaries," Molecular Endocrinology, vol. 24, no. 4, pp. 846-858, 2010.

[23] Y. Tonomoto, M. Tachibana, D. K. Dhar et al., "Differential expression of RUNX genes in human esophageal squamous cell carcinoma: downregulation of RUNX3 worsens patient prognosis," Oncology, vol. 73, no. 5-6, pp. 346-356, 2007.

[24] S. H. Lee, X. Che, J. H. Jeong et al., "Runx2 protein stabilizes hypoxia-inducible factor- $1 \alpha$ through competition with von Hippel-Lindau protein ( $\mathrm{pVHL}$ ) and stimulates angiogenesis in growth plate hypertrophic chondrocytes," The Journal of Biological Chemistry, vol. 287, pp. 14760-14771, 2012.

[25] K. Edvardsson, A. Ström, P. Jonsson, J. A. Gustafsson, and C. Williams, "Estrogen receptor $\beta$ induces antiinflammatory and antitumorigenic networks in colon cancer cells," Molecular Endocrinology, vol. 25, no. 6, pp. 969-979, 2011.
[26] N. S. Nevadunsky, J. S. Barbieri, J. Kwong et al., "RUNX3 protein is overexpressed in human epithelial ovarian cancer," Gynecologic Oncology, vol. 112, no. 2, pp. 325-330, 2009.

[27] J. H. Jeong and J. Y. Choi, "Interrelationship of RUNX2 and estrogen pathway in skeletal tissues," BMB Reports, vol. 44, pp. 613-618, 2011.

[28] Z. L. Duan, Z. L. Peng, and Z. H. Wang, "Expression and involved signal transduction pathway of autophagy gene Beclin 1 in epithelial ovarian cancer," Sichuan Da Xue Xue Bao Yi Xue Ban, vol. 38, no. 2, pp. 239-242, 2007.

[29] S. Chakrabarty and L. Kondratick, "Insulin-like growth factor binding protein-2 stimulates proliferation and activates multiple cascades of the mitogen-activated protein kinase pathways in NIH-OVCAR3 human epithelial ovarian cancer cells," Cancer Biology and Therapy, vol. 5, no. 2, pp. 189-197, 2006. 


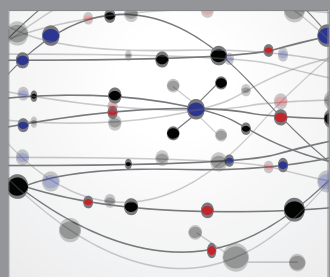

The Scientific World Journal
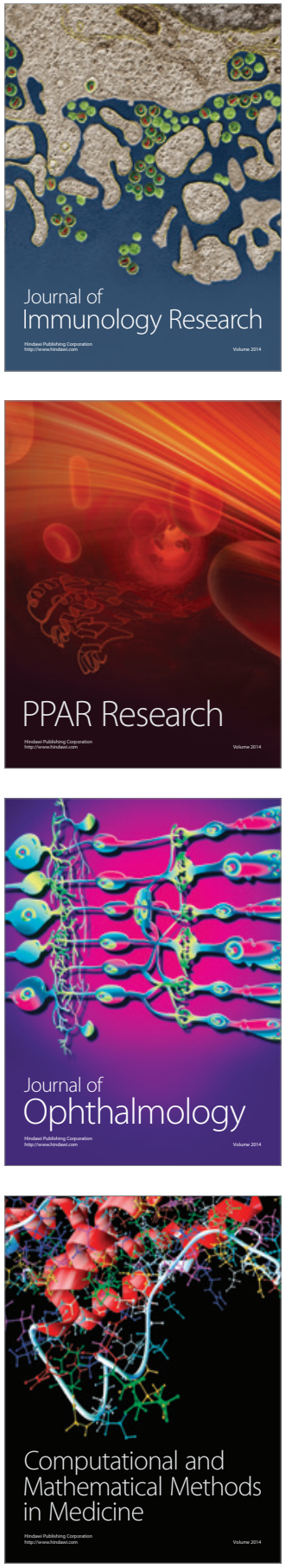

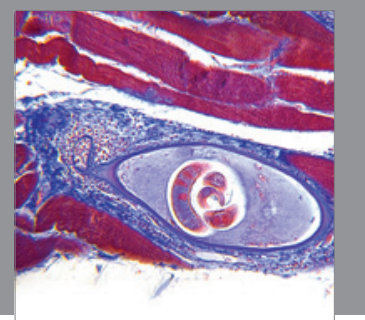

Gastroenterology

Research and Practice
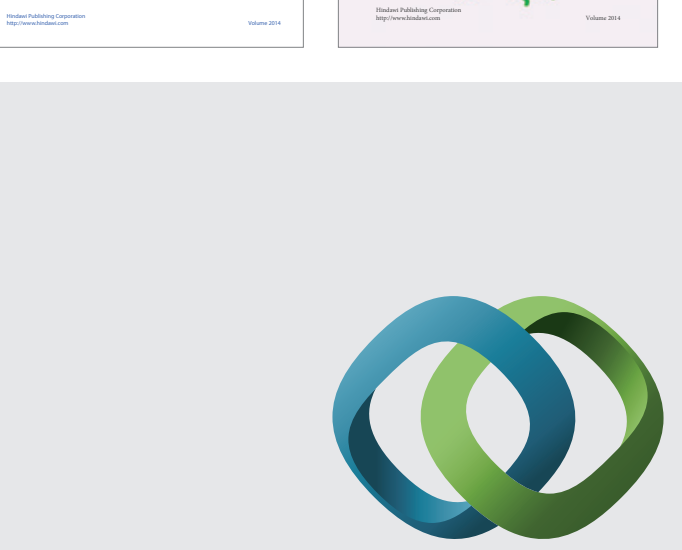

\section{Hindawi}

Submit your manuscripts at

http://www.hindawi.com
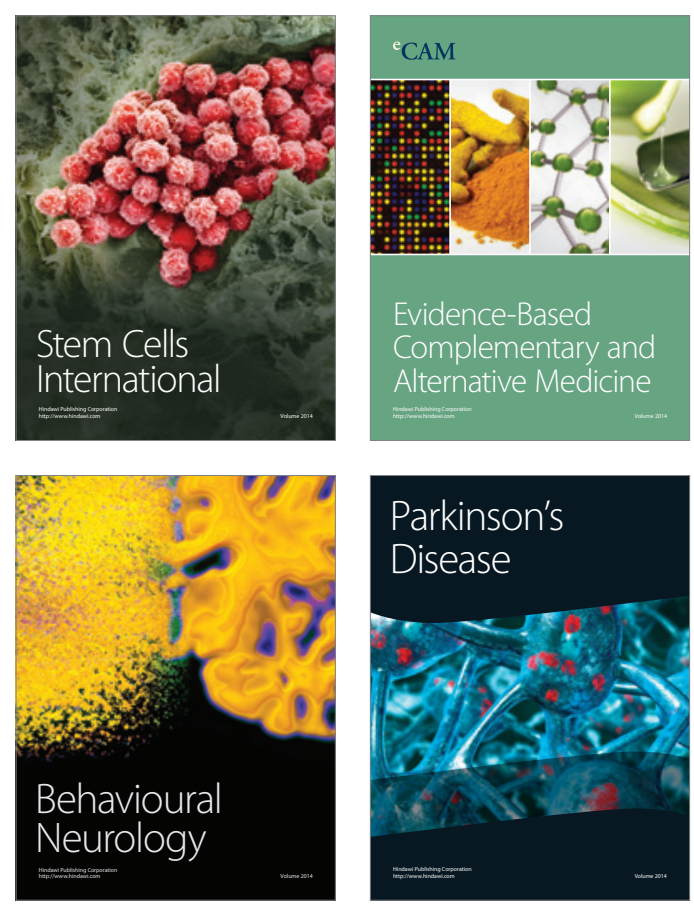

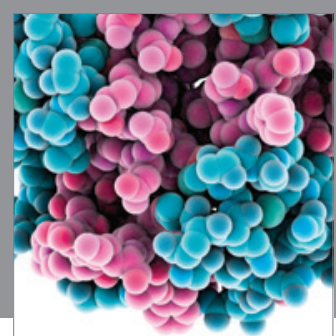

Journal of
Diabetes Research

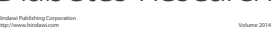

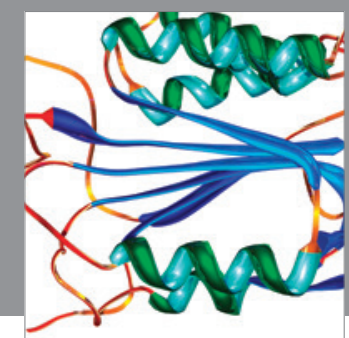

Disease Markers
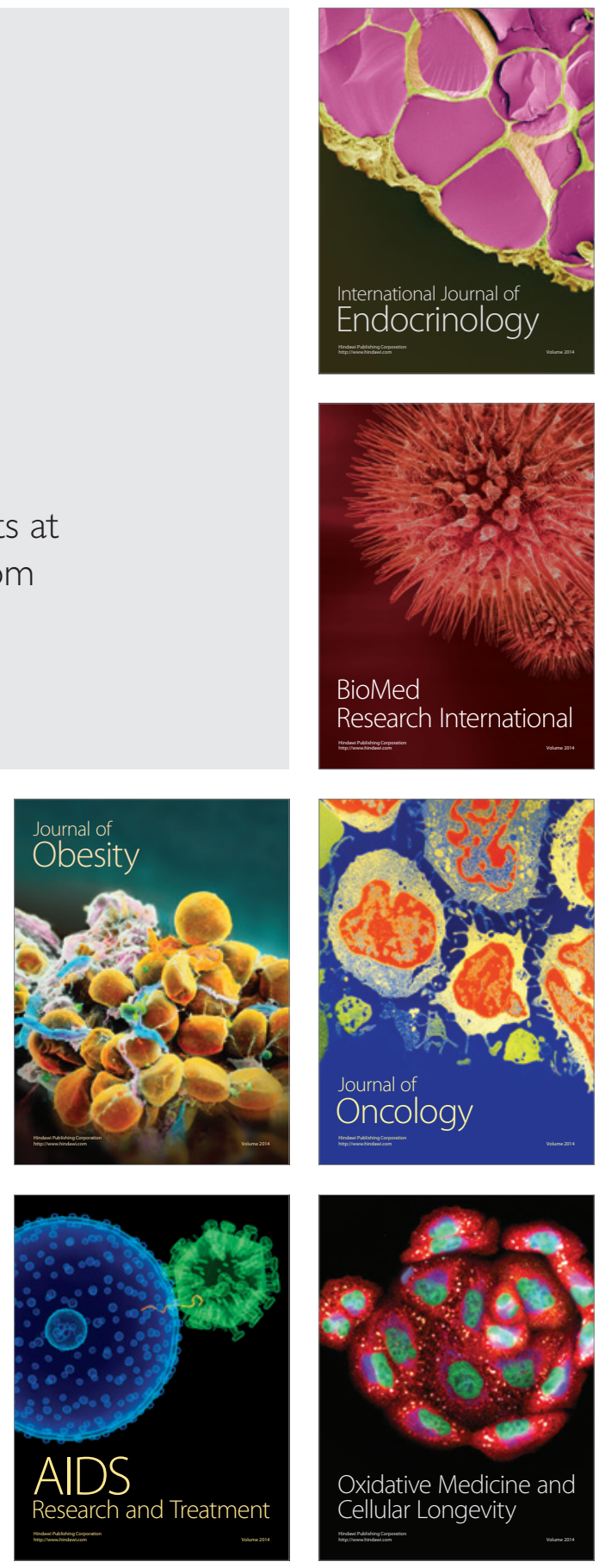